\title{
Adequabilidade do Método do Consumo Máximo Provável para o dimensionamento de sistemas prediais de água fria
}

\section{Ana Carolina Ferreira ${ }^{1}$, Pedro Henrique Rodrigues de Abreu', Mateus Alexandre da Silva², Michael Silveira Thebaldi $^{2}, *$ e Marina Neves Merlo ${ }^{2}$}

\author{
${ }^{1}$ Universidade Federal de Lavras. Departamento de Engenharia. Campus \\ Universitário. Caixa Postal 3037. Lavras-MG, Brasil (CEP 37200-000). \\ ${ }^{2}$ Universidade Federal de Lavras. Departamento de Recursos Hídricos. Campus \\ Universitário. Caixa Postal 3037. Lavras-MG, Brasil (CEP 37200-000). E-mail: \\ michael.thebaldi@ufla.br.
}

Resumo. 0 Método do Consumo Máximo Provável é comumente empregado para o dimensionamento de instalações prediais de água fria por meio de coeficientes probabilísticos para estimar a vazão provável nos trechos do sistema em função dos aparelhos que são abastecidos por cada nó, era preconizado pela ABNT NBR 5626:1998, mas não mais em sua nova edição de 2020. No entanto, de acordo com a referida norma, métodos probabilísticos ainda podem ser utilizados, desde que o dimensionamento do sistema respeite as diretrizes de funcionamento hidráulico estabelecidas. Assim, objetivou-se analisar o funcionamento hidráulico de um sistema predial de água fria, a fim de verificar a adequabilidade do Método de Dimensionamento do Consumo Máximo Provável aos novos parâmetros de projeto e funcionamento hidráulico determinados na ANBT NBR 5626:2020, especificamente em relação às pressões mínimas e à variação da carga de pressão dinâmica nos chuveiros em condição de operação isolada, e em operação concomitante com diferentes aparelhos. O limite de redução de carga de pressão dinâmica, preconizado pela nova edição da norma foi excedido apenas para duas das cinco combinações em um dos dois banheiros analisados neste estudo, porém ao se considerar a vazão de projeto como apenas a vazão do chuveiro. Desta forma, para a edificação estudada, unifamiliar típica com apenas um pavimento, o Método do Consumo Máximo Provável pôde ser utilizado, com a ressalva que sejam verificadas as cargas de pressão dinâmica nos pontos mais desfavoráveis da edificação.

Palavras-chave: Aparelhos sanitários; Carga de pressão dinâmica; Dimensionamento; Desempenho hidráulico; Sistemas hidráulicos prediais.
Recebido

20/08/2021

Aceito

$15 / 12 / 2021$

Publicado

31/12/2021

Acesso aberto

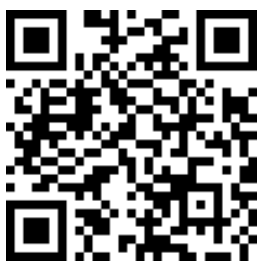

ORCID

(D) 0000-0002-5043-0178 Ana Carolina Ferreira

(1) 0000-0001-9425-7533 Pedro Henrique Rodrigues de Abreu

(D) 0000-0002-2849-0668 Mateus Alexandre da Silva

D) 0000-0002-4579-6714

Michael Silveira

Thebaldi

ISSN 2359-1412/RBGAS-2021-0106/2021/8/20/23/1633

Rev. Bras. Gest. Amb. Sustent.

http://revista.ecogestaobrasil.net 
Abstract. Suitability of the Maximum Probable Consumption Method for the design of potable water building systems. The Maximum Probable Consumption Method is commonly used for designing building potable water supply installations by probabilistic coefficients to estimate the probable flow in the system sections depending on the devices that are supplied by each node, was recommended by ABNT NBR 5626:1998 but no longer by its new edition, from 2020. However, according to that standard, probabilistic methods can still be used since the system design respects the established hydraulic operation guidelines. Thus, the objective was to analyze the hydraulic performance of a building potable water supply system, to verify the suitability of the Maximum Probable Consumption design method to the new design and hydraulic performance parameters determined in ANBT NBR 5626:2020, specifically concerning the minimum pressures and the variation of the dynamic pressure in the showers in isolated operation condition and due to simultaneous operation with different devices. The dynamic pressure head reduction limit, recommended by the standard new edition was exceeded only for two of the five combinations in one of the two analyzed bathrooms in this study, however when considering the design flow as only the shower flow. Thus, for the studied building, a typical single-family with only one floor, the Maximum Probable Consumption Method could be used, with the proviso that only the dynamic pressure head in the most unfavorable points of the building were verified.

Keywords: Sanitary fixtures; Dynamic pressure head; Hydraulic design; Hydraulic performance; Building water supply systems.
(D) $0000-0002-9518-6033$

Marina Neves Merlo

\section{Introdução}

Os sistemas de instalações hidráulicas prediais estão em constante evolução, devido ao desenvolvimento e utilização de novos materiais e técnicas, contudo, de acordo com o SINDUSCON-SP, $75 \%$ das patologias em edificações estão relacionadas às instalações prediais de água e esgoto (Carvalho Júnior, 2015), sendo parte destas geradas durante a fase de dimensionamento do sistema (Ilha, 2009).

Além dos problemas relacionados ao uso do sistema, o dimensionamento inadequado, em caso de superdimensionamento, pode gerar custos desnecessários (Silva et al., 2019), seja ao adquirir os materiais a serem utilizados para a sua implantação ou mesmo o maior consumo de água durante a sua vida útil. Ainda neste sentido, o superdimensionamento pode ser afetar a qualidade da água nos pontos de utilização devido ao aumento do tempo de residência da água no sistema, propiciando o crescimento de microrganismos, como a Legionella pneumophila (Mangalenkar e Gumaste, 2021).

Para mais, a demanda pela água em edificações residenciais é uma variável com um grande fator probabilístico e de incerteza, porém, de determinação essencial para o dimensionamento dos sistemas prediais de água potável. A estimativa da demanda de água é difícil devido ao número de fatores que a afetam e a natureza dinâmica da própria variável, ligada ao uso dos vários aparelhos sanitários presentes no sistema (Mangalenkar e Gumaste, 2021). 
Com o objetivo de evitar tais problemas, além de padronizar diretrizes e exigências, garantindo a qualidade e bom desempenho das instalações hidráulicas prediais, existe uma norma para regulamentá-las. A norma ABNT NBR 5626:2020 (ABNT, 2020) é a versão mais recente utilizada para instalações prediais de água fria e água quente.

Em sua versão anterior, a norma ABNT NBR 5626:1998 (ABNT, 1998) preconizava que o dimensionamento do sistema fosse realizado por meio do Método do Consumo Máximo Provável, que utiliza um coeficiente probabilístico para estimar a vazão provável nos trechos do sistema em função dos aparelhos que são abastecidos por cada nó, o que não foi mantido na nova edição de 2020. No entanto, a nova edição permite o uso de métodos probabilísticos, desde que o dimensionamento do sistema respeite as diretrizes de funcionamento hidráulico estabelecidas e seja reconhecido ou devidamente fundamentado.

Diferentemente da versão anterior de 1998, a norma ABNT NBR 5626:2020 (ABNT, 2020) faz algumas exigências específicas, como que a redução máxima de pressão dinâmica no chuveiro ao se abrir qualquer outro ponto de utilização não deve ser maior do que $10 \%$ do valor da pressão desse funcionando com a sua vazão de projeto.

Tem-se que o correto dimensionamento de sistemas de engenharia, a partir de técnicas e diretrizes compatíveis com seus usos preponderantes e funcionamento, está intimamente ligado com o desenvolvimento sustentável. Em uma primeira aproximação, vê-se a utilização de material estritamente necessário para a execução dos empreendimentos e, obviamente, o não desperdício de recursos financeiros. No caso de sistemas hidráulicos há além o uso racional de recurso hídrico e energético, premissas estas determinadas para concepção de sistemas hidráulicas de água fria e quente (ABNT, 2020). Assim, objetivou-se com este trabalho verificar a adequabilidade do Método do Consumo Máximo Provável ao exigido pela norma ABNT NBR 5626:2020 (ABNT, 2020), analisando as cargas de pressão dinâmicas nos chuveiros da edificação face ao uso simultâneo desses com diferentes aparelhos sanitários, em relação ao uso isolado dos chuveiros e ao funcionamento com a vazão de projeto calculada pelo Método do Consumo Máximo Provável.

\section{Material e métodos}

\section{água fria \\ Caracterização da edificação estudada e diretrizes de traçado da rede de \\ 0 projeto analisado neste estudo trata-se de uma edificação de um pavimento, com} 198,57 m² distribuídos entre os ambientes: depósito, suíte, área de serviço, lavabo, dois banheiros, dois dormitórios, cozinha, despensa, sala de estar, escritório e garagem. Foi estabelecido um reservatório de água fria, instalado na parte superior da edificação, a 0,80 m de altura a partir da laje. A planta baixa da edificação pode ser vista na Figura 1.

0 local de instalação do reservatório de água na cobertura foi definido levando em consideração a localização da maior parte dos aparelhos hidráulicos, ou seja, acima do banheiro da suíte e do lavabo. Posteriormente, foram determinados os locais de saída dos barriletes e o posicionamento das colunas de distribuição. Para cada ambiente que necessitava de abastecimento de água, foi atribuída uma coluna de distribuição, sendo eles, lavanderia, lavabo, cada um dos banheiros e cozinha. Cada coluna de distribuição abasteceu determinados aparelhos sanitários, conforme disposto na Tabela 1. 


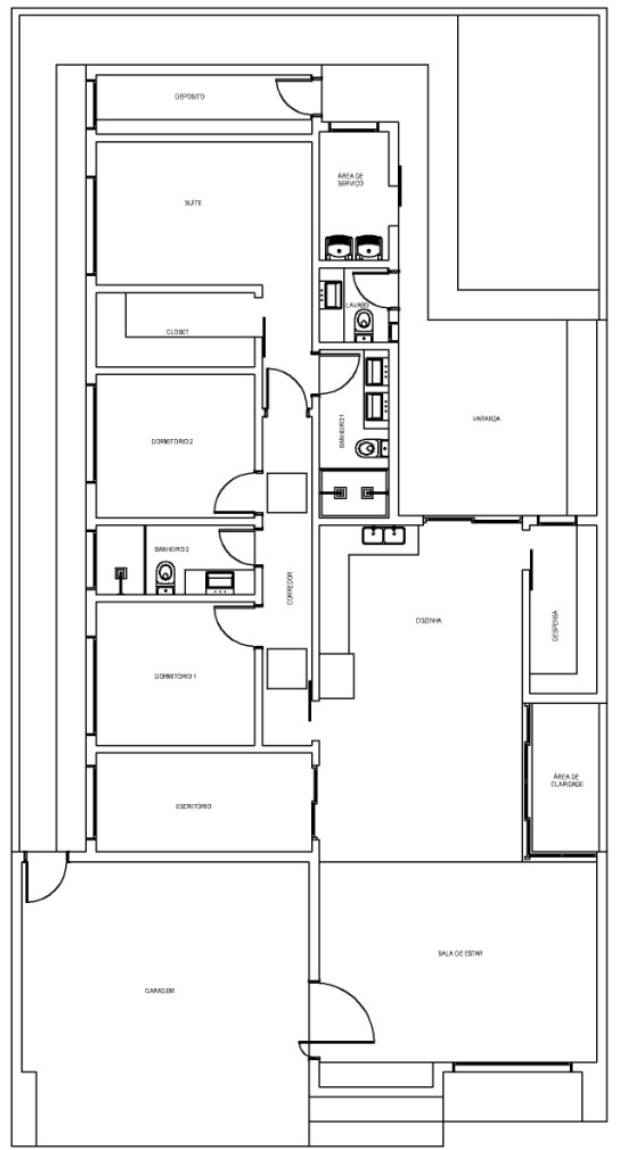

Figura 1. Planta baixa da edificação analisada.

Tabela 1. Colunas de distribuição e seus respectivos aparelhos sanitários.

\begin{tabular}{|c|c|}
\hline Colunas de distribuição & Aparelhos sanitários \\
\hline 1 & TQ e MLR \\
\hline 2 & LV, DH e BCA \\
\hline 3 & $2 \mathrm{LV}, \mathrm{DH}, \mathrm{BCA}$ e CH \\
\hline 4 & PIA e 2 BE \\
\hline 5 & LV, DH, BCA e CH \\
\hline
\end{tabular}

Legenda: $\mathrm{TQ}=$ Tanque; $M L R=$ Máquina de lavar roupa; $\mathrm{LV}=$ Lavatório; $\mathrm{DH}=$ Ducha higiênica; $\mathrm{BCA}$ = Bacia sanitária com caixa acoplada; $\mathrm{CH}=$ Chuveiro; PIA = Pia; $\mathrm{BE}=$ Bebedouro.

A localização do reservatório, o traçado dos barriletes, bem como o posicionamento das colunas de distribuição,são mostrados na Figura 2. 


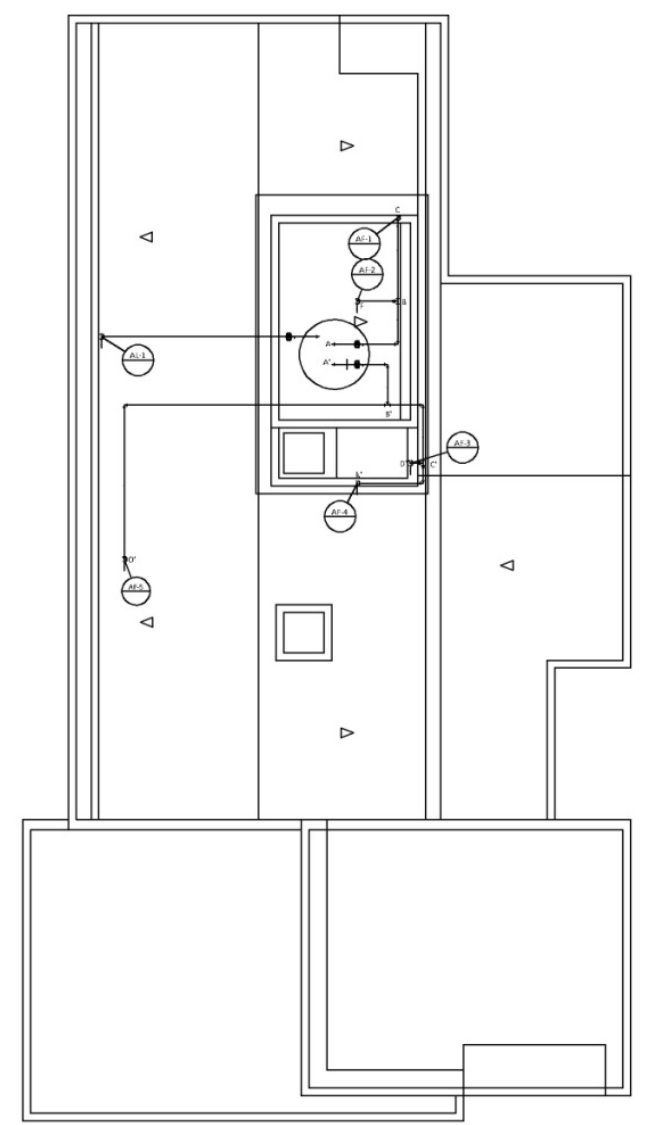

Figura 2. Localização do reservatório, traçado dos barriletes e posicionamento das colunas de distribuição.

Após a definição do posicionamento das colunas de distribuição, iniciou-se o traçado dos ramais. Com os ramais distribuídos de maneira satisfatória, levando em consideração os aspectos arquitetônicos e estruturais da edificação, os sub-ramais foram traçados, ligando os ramais horizontais a cada aparelho sanitário. Por fim, foram posicionados no início de cada coluna de distribuição um registro de gaveta e acessórios onde havia necessidade. As perspectivas isométricas da instalação hidráulica do reservatório, banheiro 1 e banheiro 2, estão mostradas na Figura 3.

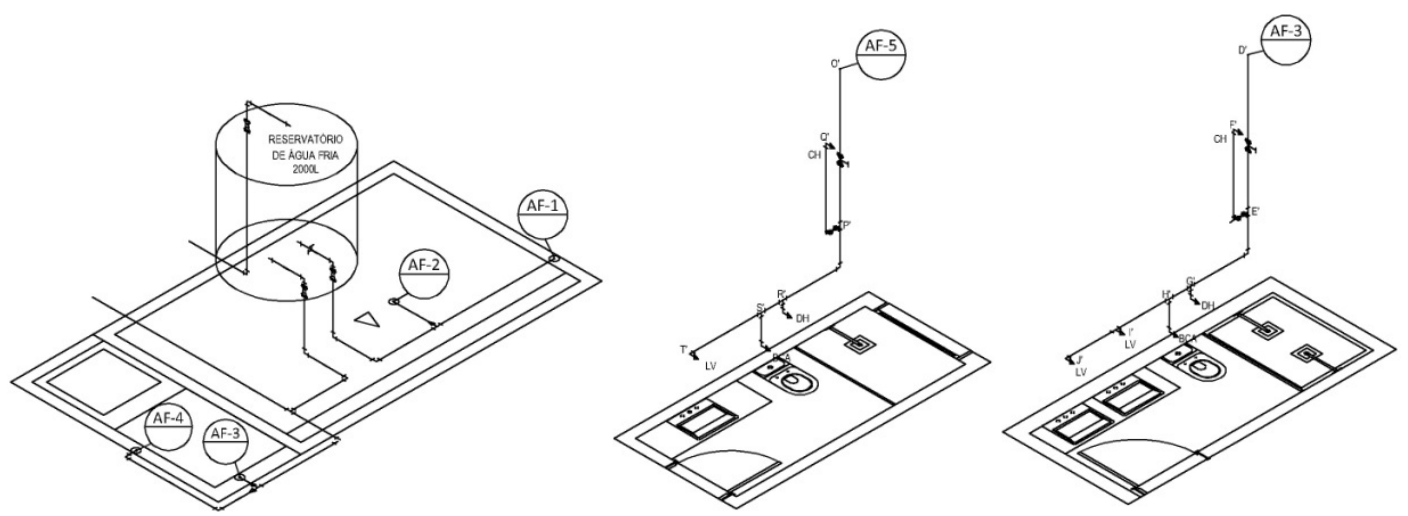

Figura 3. Perspectivas isométricas da instalação hidráulica do reservatório (a esquerda), banheiro 1 (ao centro) e banheiro 2 (a direita). 
Já na Figura 4 são mostradas as perspectivas isométricas da instalação hidráulica da cozinha, lavabo e área de serviço.
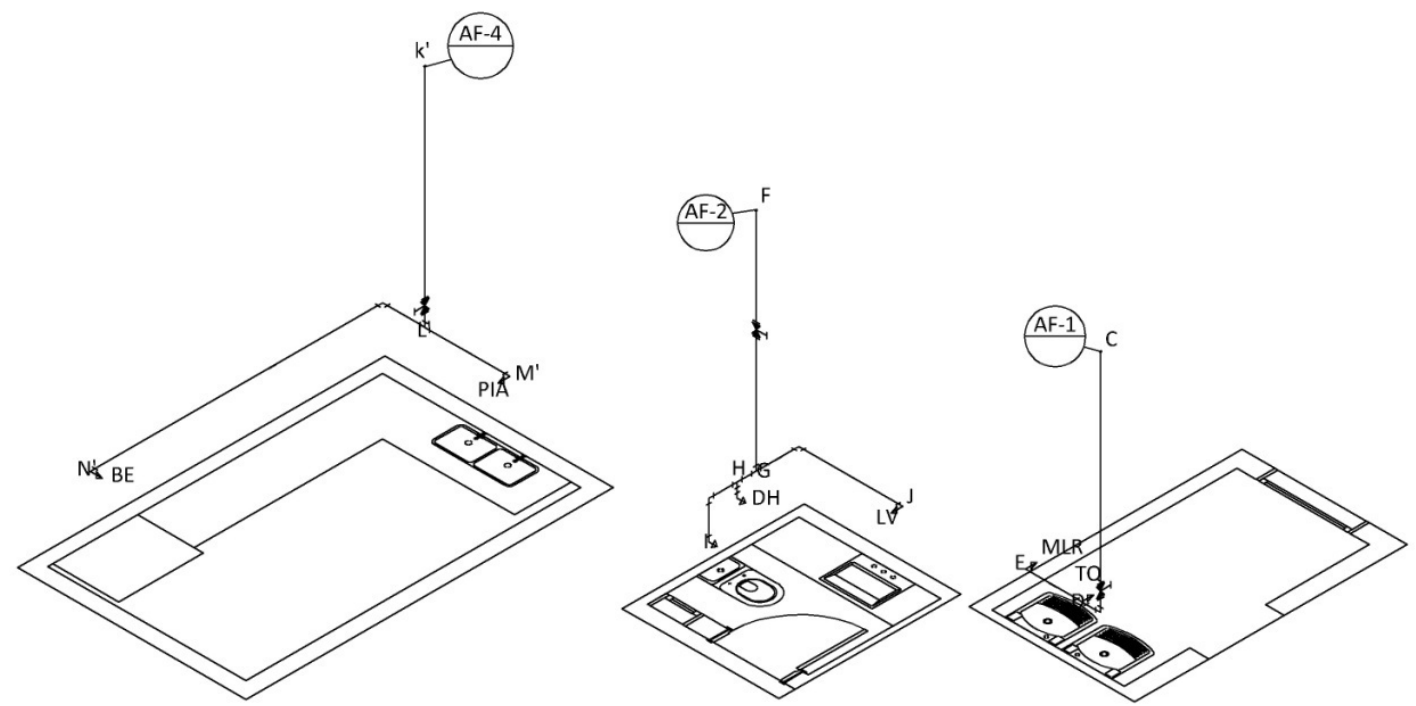

Figura 4. Perspectivas isométricas da instalação hidráulica da cozinha (a esquerda), lavabo (ao centro) e área de serviço (a direita).

\section{Dimensionamento por meio do Método do Consumo Máximo Provável}

Para o dimensionamento das tubulações, primeiramente foi obtido o somatório dos pesos relativo dos equipamentos e posteriormente foram determinadas as vazões e os diâmetros nominais (DN) de cada trecho do sistema. Para tal, utilizou-se a tabela de pesos relativos que relaciona o aparelho sanitário e sua respectiva peça de utilização, disponível em Azevedo Netto e Fernández y Fernández (2015).

Em seguida, para obter o diâmetro interno da tubulação (Di) foi utilizado o nomograma de pesos, vazões e diâmetros, apresentado em Botelho e Ribeiro Júnior (2014), acumulando em cada trecho do sistema os pesos relativos dos equipamentos abastecidos por esses.

Após determinação dos diâmetros internos, calculou-se a área da seção da tubulação de cada trecho. Em seguida foram calculadas as velocidades por meio da Equação 1.

$$
\mathrm{V}=\frac{\mathrm{Q}}{\mathrm{A}}
$$

Equação 1

Em que: $\mathrm{V}=$ velocidade $\left(\mathrm{m} \mathrm{s}^{-1}\right), \mathrm{Q}=$ vazão $\left(\mathrm{m}^{3} \mathrm{~s}^{-1}\right)$, e $\mathrm{A}=$ área $\left(\mathrm{m}^{2}\right)$.

As velocidades foram verificadas para que não excedessem o valor de $3 \mathrm{~m} \mathrm{~s}^{-1}$, objetivando a diminuição dos picos de sobrepressão no sistema, conforme sugerido na norma ABNT NBR 5626:2020 (ABNT, 2020). Determinados os diâmetros e vazões de cada 
trecho, pôde-se iniciar o cálculo da perda de carga utilizando o Método dos Comprimentos Virtuais.

\section{Perda de carga e verificação de funcionamento hidráulico}

Para o cálculo dos comprimentos virtuais, foi necessária a medição em planta do comprimento real de cada trecho, nos quais posteriormente foi adicionado o comprimento equivalente de todas as peças correspondentes a cada trecho, comprimento esses dispostos em razão do número de diâmetros da tubulação em Azevedo Netto e Fernández (2015). Com isso, foi possível determinar o comprimento virtual da tubulação (Equação 2).

$$
\mathrm{L}_{\mathrm{v}}=\mathrm{L}+\mathrm{L}_{\mathrm{eq}}
$$

Equação 2

Em que: $\mathrm{L}_{\mathrm{v}}=$ comprimento virtual $(\mathrm{m}), \mathrm{L}=$ comprimento medido em planta $(\mathrm{m})$, e $\mathrm{L}_{\mathrm{eq}}=$ comprimento equivalente às peças especiais $(\mathrm{m})$.

Posteriormente, o cálculo da perda de carga de cada trecho foi feito por meio da Equação de Flamant para condução água fria em tubos de PVC (Equação 3). Conforme indicado pela norma ABNT NBR 15704-1:2011 (ABNT, 2011), nos trechos onde havia registros de pressão, a perda de carga desse foi contabilizada separadamente, por meio da Equação 4. As perdas de carga obtidas referentes aos registros de pressão foram posteriormente somadas à perda de seus respectivos trechos (Equação 5). Por fim, analisou-se a pressão dinâmica disponível nos chuveiros, visto que esses são os pontos mais desfavoráveis da instalação hidráulica predial analisada, utilizando a Equação 6.

$$
\begin{gathered}
\mathrm{h}_{\mathrm{f}}=0,000824 \cdot \frac{\mathrm{Q}^{1,75}}{\mathrm{Di}^{4,75}} \cdot \mathrm{L}_{\mathrm{v}} \\
\Delta_{\mathrm{h}}=8 \cdot 10^{5} \cdot \mathrm{K} \cdot \mathrm{Q}^{2} \cdot \mathrm{\pi}^{-2} \cdot \mathrm{d}^{-4} \\
\Delta \mathrm{H}=\mathrm{h}_{\mathrm{f}}+\Delta_{\mathrm{h}} \\
\mathrm{P}_{\mathrm{d}}=\mathrm{P}_{\mathrm{e}}-\sum \Delta \mathrm{H}
\end{gathered}
$$

Equação 3

Equação 4

Equação 5

Equação 6

Em que: $\mathrm{h}_{\mathrm{f}}=$ perda de carga (m.c.a.), Di = diâmetro interno do tubo (m), $\Delta_{\mathrm{h}}=$ perda de carga no registro de pressão (m.c.a.), $\mathrm{K}=$ coeficiente de perda de carga no registro (vide a norma ABNT NBR 15704-1/2011 - adotou-se o valor de $\mathrm{K}=40$ e 32 para DN20 e DN25, respectivamente), $\mathrm{d}=$ diâmetro interno do tubo $(\mathrm{mm}), \Delta \mathrm{H}=$ perda de carga total no trecho (m.c.a.), $\mathrm{P}_{\mathrm{d}}=$ carga de pressão dinâmica (m.c.a.), $\mathrm{P}_{\mathrm{e}}=$ carga pressão estática (m.c.a.), e $\sum \Delta \mathrm{H}=$ somatório das perdas de carga até o chuveiro (m.c.a.).

Após o dimensionamento, foram realizadas combinações a fim de analisar o funcionamento simultâneo dos chuveiros com os demais aparelhos do sistema, verificando a pressão dinâmica encontrada em cada combinação. Para isso, o cálculo da perda de carga 
foi realizado em cada trecho utilizando o somatório das vazões de projeto desses, disponíveis em Azevedo Netto e Fernández (2015), de acordo com os aparelhos utilizados em cada combinação. A porcentagem de variação da pressão foi determinada tomando como referência a pressão dinâmica do chuveiro, com vazão de projeto, operando isoladamente.

As combinações do funcionamento simultâneo para o chuveiro do banheiro 1 e o chuveiro do banheiro 2, sendo esse último acompanhado pela indicação de linha ('), são mostradas na Tabela 2.

Tabela 2. Combinações de funcionamento simultâneo para o chuveiro dos banheiros 1 e 2 , sendo esse último acompanhado pela indicação de linha (').

\begin{tabular}{|c|c|}
\hline Combinações & Aparelhos sanitários \\
\hline A e A $^{\prime}$ & CH e LV \\
\hline B e B' & CH e BCA \\
\hline C e C' $^{\prime}$ & CH e DH \\
\hline D e D' $^{\prime}$ & CH e CH \\
\hline E eE' $^{\prime}$ & CH e PIA \\
\hline
\end{tabular}

Legenda: LV = Lavatório; $\mathrm{DH}=$ Ducha higiênica; $\mathrm{BCA}=$ Bacia sanitária com caixa acoplada; $\mathrm{CH}=$ Chuveiro; PIA = Pia.

\section{Resultados e discussão}

Ao analisar os pontos mais desfavoráveis da edificação (neste caso, os chuveiros), verificou-se que as cargas pressão dinâmica encontradas $(0,205$ e $-0,897$ m.c.a. para os chuveiros do banheiro 1 e 2, respectivamente) não satisfizeram os requisitos da norma ABNT NBR 5626:2020 (ABNT, 2020), que determina que a pressão dinâmica da água nos pontos de utilização não pode ser inferior a $10 \mathrm{kPa}$ (utilizando o valor da aceleração da gravidade de $9,81 \mathrm{~m} \mathrm{~s}^{-2}$, tem-se 1,02 m.c.a.). Desta forma, foi necessário realizar o redimensionamento nos trechos que abastecem os chuveiros, mantendo fixa a altura do reservatório em $0,80 \mathrm{~m}$ e alterando os valores de diâmetro, aumentando-os e respeitando os diâmetros comerciais, de montante à jusante, partindo do barrilete que se origina no reservatório, a fim de atender a norma. Redimensionamentos não são práticas exclusivamente adotadas em dimensionamentos "manuais" como realizado neste estudo. Tal fato é constatado por Oliveira (2015), em que, apesar da utilização de um software para o dimensionamento da instalação hidráulica predial, foi necessário um redimensionamento do sistema após o dimensionamento inicial para que as cargas de pressão dinâmica atendessem a norma vigente.

As mudanças ocorreram nos trechos A'-B' (DN25 para DN 40), B'-C', C'-D', D'-E (DN 25 para DN 32), B'-O' (DN 20 para DN 32), O'-P' e P'-Q' (DN 20 para DN 25). Os valores das cargas de pressão dinâmica em cada chuveiro da edificação, obtidas após o redimensionamento, estão expressos na Tabela 3.

Tabela 3. Cargas de pressão dinâmica nos chuveiros do banheiro 1 e 2, obtidas após o redimensionamento do sistema.

\begin{tabular}{|c|c|}
\hline Ambiente & P $_{\mathbf{d}}$ (m.c.a.) \\
\hline A e A' $^{\prime}$ & 1,06 \\
\hline B e B' $^{\prime}$ & 1,25 \\
\hline
\end{tabular}


Apesar da água percorrer uma maior extensão de tubulação para abastecer o chuveiro do banheiro $2(24,0 \mathrm{~m})$ em relação ao do banheiro $1(18,03 \mathrm{~m})$ e de possuir trechos com menores diâmetros, o chuveiro do banheiro 1 apresentou uma menor carga de pressão dinâmica. Sabe-se que os registros de pressão são responsáveis por uma alta perda de carga. No caso avaliado, para chegar até os chuveiros do sistema, a água passa por um registro de pressão que, para o banheiro 1, possui o diâmetro nominal de $20 \mathrm{~mm}$ e, para o banheiro 2, de $25 \mathrm{~mm}$. Sendo assim, a menor pressão encontrada para o chuveiro do banheiro 1, apesar do menor comprimento virtual da tubulação, é explicada pelo menor diâmetro do registro de pressão, que, em combinação com a vazão escoada, proporcionou uma maior perda de carga. Para efeito de comparação, a norma britânica BS 6700 (BSI, 2006), que regulamenta o abastecimento de água doméstico, sugere que o diâmetro do sub-ramal do chuveiro não seja menor que $25 \mathrm{~mm}$ (fato ocorrido para o banheiro 1), minimizando, desta forma, o risco de queimaduras caso o abastecimento de água falhe, em função da menor redução da carga de pressão dinâmica disponível e consequentemente, maior vazão no aparelho sanitário.

Os valores das cargas de pressão dinâmicas, bem como as demais variáveis hidráulicas obtidas considerando o uso individual dos chuveiros com vazão de projeto dos banheiros 1 e 2 podem ser vistos na Tabela 4 .

Tabela 4. Cargas de pressão dinâmica e variáveis hidráulicas obtidas para os chuveiros dos banheiros 1 e 2, considerando o seu uso individual com vazão de projeto.

\begin{tabular}{|c|c|c|c|c|c|c|c|}
\hline Ambiente & Trecho & $\begin{array}{c}Q \\
\left(\mathbf{L} \mathbf{s}^{-1}\right)\end{array}$ & $\begin{array}{c}\text { DN } \\
(\mathrm{mm})\end{array}$ & $\begin{array}{c}\mathrm{Di} \\
(\mathrm{mm})\end{array}$ & $\begin{array}{l}\text { Lv } \\
\text { (m) }\end{array}$ & $\Delta H(m . c . a)$ & $\begin{array}{c}\text { Pd } \\
\text { (m.c.a.) }\end{array}$ \\
\hline \multirow{5}{*}{ Banheiro 1} & $A^{\prime}-B^{\prime}$ & 0,10 & 40 & 35,2 & 7,01 & 0,005 & - \\
\hline & $B^{\prime}-C^{\prime}$ & 0,10 & 32 & 27,8 & 4,49 & 0,009 & - \\
\hline & $\mathrm{C}^{\prime}-\mathrm{D}^{\prime}$ & 0,10 & 32 & 27,8 & 0,25 & 0,001 & - \\
\hline & $\mathrm{D}^{\prime}-\mathrm{E}^{\prime}$ & 0,10 & 32 & 27,8 & 3,71 & 0,008 & - \\
\hline & $E^{\prime}-F^{\prime}$ & 0,10 & 20 & 17,0 & 2,58 & 0,403 & 1,27 \\
\hline \multirow{4}{*}{ Banheiro 2} & $A^{\prime}-B^{\prime}$ & 0,10 & 40 & 35,2 & 7,01 & 0,005 & - \\
\hline & $\mathrm{B}^{\prime}-\mathrm{O}^{\prime}$ & 0,10 & 32 & 27,8 & 10,89 & 0,022 & - \\
\hline & $\mathrm{O}^{\prime}-\mathrm{P}^{\prime}$ & 0,10 & 25 & 21,6 & 3,17 & 0,021 & - \\
\hline & $P^{\prime}-Q^{\prime}$ & 0,10 & 25 & 21,6 & 2,92 & 0,127 & 1,53 \\
\hline
\end{tabular}

No uso isolado dos chuveiros, as cargas de pressão dinâmica encontradas mantiveram-se acima da carga de pressão dinâmica mínima estabelecida pela norma ABNT NBR 5626:2020 (ABNT, 2020), além de apresentarem valores maiores em relação ao encontrado utilizando a vazão obtida por meio do Método do Consumo Máximo Provável. Pelo fato de ser utilizada uma vazão de projeto para o chuveiro de $0,1 \mathrm{~L} \mathrm{~s}^{-1}$, menor do que as consideradas em trânsito pelos trechos do sistema para o Método do Consumo Máximo Provável, a perda de carga em cada trecho apresentou valores inferiores. Assim, o menor valor de perda de carga resultou em um maior valor de pressão dinâmica esperada no ponto de saída dos chuveiros.

Na Tabela 5 estão apresentados os valores das pressões dinâmicas para cada combinação de uso simultâneo para o chuveiro do banheiro 1. 
Tabela 5. Cargas de pressão dinâmica e variáveis hidráulicas obtidas para as combinações de operação simultânea para o chuveiro do banheiro 1.

\begin{tabular}{|c|c|c|c|c|c|c|c|}
\hline Combinação & Trecho & $\begin{array}{c}Q \\
(L-1)\end{array}$ & $\begin{array}{c}\mathrm{DN} \\
(\mathrm{mm})\end{array}$ & $\begin{array}{c}\mathrm{Di} \\
(\mathrm{mm})\end{array}$ & $\begin{array}{c}\text { Lv } \\
(\mathrm{m})\end{array}$ & $\begin{array}{c}\Delta H \\
\text { (m.c.a) }\end{array}$ & $\begin{array}{c}\text { Pd } \\
\text { (m.c.a.) }\end{array}$ \\
\hline \multirow{5}{*}{ A } & $A^{\prime}-B^{\prime}$ & 0,25 & 40 & 35,2 & 7,01 & 0,023 & - \\
\hline & $\mathrm{B}^{\prime}-\mathrm{C}^{\prime}$ & 0,25 & 32 & 27,8 & 4,49 & 0,045 & - \\
\hline & $C^{\prime}-D^{\prime}$ & 0,25 & 32 & 27,8 & 0,25 & 0,003 & - \\
\hline & $D^{\prime}-E^{\prime}$ & 0,25 & 32 & 27,8 & 3,71 & 0,037 & - \\
\hline & $E^{\prime}-F^{\prime}$ & 0,10 & 20 & 17,0 & 2,58 & 0,406 & 1,19 \\
\hline \multirow{5}{*}{ B } & $A^{\prime}-B^{\prime}$ & 0,25 & 40 & 35,2 & 7,01 & 0,023 & - \\
\hline & $B^{\prime}-C^{\prime}$ & 0,25 & 32 & 27,8 & 4,49 & 0,045 & - \\
\hline & $C^{\prime}-D^{\prime}$ & 0,25 & 32 & 27,8 & 0,25 & 0,003 & - \\
\hline & $D^{\prime}-E^{\prime}$ & 0,25 & 32 & 27,8 & 3,71 & 0,037 & - \\
\hline & $E^{\prime}-F^{\prime}$ & 0,10 & 20 & 17,0 & 2,58 & 0,406 & 1,19 \\
\hline \multirow{5}{*}{$\mathrm{C}$} & $A^{\prime}-B^{\prime}$ & 0,20 & 40 & 35,2 & 7,01 & 0,016 & - \\
\hline & $B^{\prime}-C^{\prime}$ & 0,20 & 32 & 27,8 & 4,49 & 0,031 & - \\
\hline & $C^{\prime}-D^{\prime}$ & 0,20 & 32 & 27,8 & 0,25 & 0,002 & - \\
\hline & $D^{\prime}-E^{\prime}$ & 0,20 & 32 & 27,8 & 3,71 & 0,025 & - \\
\hline & $E^{\prime}-F^{\prime}$ & 0,10 & 20 & 17,0 & 2,58 & 0,403 & 1,22 \\
\hline \multirow{5}{*}{$\mathrm{D}$} & $A^{\prime}-B^{\prime}$ & 0,20 & 40 & 35,2 & 7,01 & 0,016 & - \\
\hline & $B^{\prime}-C^{\prime}$ & 0,10 & 32 & 27,8 & 4,49 & 0,009 & - \\
\hline & $C^{\prime}-D^{\prime}$ & 0,10 & 32 & 27,8 & 0,25 & 0,001 & - \\
\hline & $D^{\prime}-E^{\prime}$ & 0,10 & 32 & 27,8 & 3,71 & 0,008 & - \\
\hline & $E^{\prime}-F^{\prime}$ & 0,10 & 20 & 17,0 & 2,58 & 0,403 & 1,26 \\
\hline \multirow{5}{*}{$\mathrm{E}$} & $A^{\prime}-B^{\prime}$ & 0,35 & 40 & 35,2 & 7,01 & 0,041 & - \\
\hline & $B^{\prime}-C^{\prime}$ & 0,35 & 32 & 27,8 & 4,49 & 0,082 & - \\
\hline & $C^{\prime}-D^{\prime}$ & 0,10 & 32 & 27,8 & 0,25 & 0,001 & - \\
\hline & $D^{\prime}-E^{\prime}$ & 0,10 & 32 & 27,8 & 3,71 & 0,008 & - \\
\hline & $E^{\prime}-F^{\prime}$ & 0,10 & 20 & 17,0 & 2,58 & 0,403 & 1,17 \\
\hline
\end{tabular}

Observou-se, por meio da análise da Tabela 5, que a menor carga de pressão dinâmica ocorreu na combinação E. Uma vez que os valores de diâmetros são constantes em todas as simulações, chega-se à conclusão que os valores de perda de carga mais elevados e, consequentemente, a menor carga de pressão dinâmica deve-se à utilização concomitante com a pia, a qual possui vazão de projeto maior em relação aos aparelhos considerados nas demais combinações, além de um maior comprimento de tubulação para que seja abastecida em relação aos aparelhos localizados à jusante do chuveiro do banheiro 1, para a instalação com concepção estudada.

No mesmo sentido, a maior carga de pressão dinâmica foi estimada para a combinação D, pois o uso simultâneo dos dois chuveiros levou a uma perda de carga menor devido ao baixo valor de vazão de projeto apresentado por tal aparelho e maiores diâmetros dos trechos que o abastecem, além de possuírem apenas um trecho de tubulação em comum ( $\left.\mathrm{A}^{\prime}-\mathrm{B}^{\prime}\right)$. Ainda, todas as combinações apresentaram menores valores de vazão em relação aos valores encontrados utilizando o Método do Consumo Máximo Provável, apresentando, consequentemente, maiores valores de carga de pressão dinâmica. De maneira análoga, Zanoni (2018) comparou a vazão obtida por meio do Método do Consumo Máximo Provável à vazão obtida por uma combinação de utilização simultânea do chuveiro, lavatório e pia da cozinha. Apesar de utilizar uma combinação de três aparelhos (um a mais em relação às combinações definidas neste estudo), a autora ainda obteve um valor de vazão menor comparado ao obtido utilizando o Método do Consumo Máximo Provável. 
Na Tabela 6 estão apresentados os valores das cargas de pressão dinâmica de cada combinação para o chuveiro do banheiro 2 .

Tabela 6. Cargas de pressão dinâmica e variáveis hidráulicas obtidas para as combinações de operação simultânea para o chuveiro do banheiro 2 .

\begin{tabular}{|c|c|c|c|c|c|c|c|}
\hline Combinação & Trecho & $\begin{array}{c}Q \\
\text { (L s-1) }\end{array}$ & $\begin{array}{c}\text { DN } \\
(\mathrm{mm})\end{array}$ & $\begin{array}{c}\text { Di } \\
(\mathbf{m m})\end{array}$ & $\begin{array}{c}\mathrm{Lv} \\
\text { (m) }\end{array}$ & $\begin{array}{c}\Delta \mathrm{H} \\
\text { (m.c.a) }\end{array}$ & $\begin{array}{c}\text { Pd } \\
\text { (m.c.a.) }\end{array}$ \\
\hline \multirow{4}{*}{$A^{\prime}$} & $A^{\prime}-B^{\prime}$ & 0,25 & 40 & 35,2 & 7,01 & 0,023 & - \\
\hline & $\mathrm{B}^{\prime}-\mathrm{O}^{\prime}$ & 0,25 & 32 & 27,8 & 10,89 & 0,110 & - \\
\hline & $\mathrm{O}^{\prime}-\mathrm{P}^{\prime}$ & 0,25 & 25 & 21,6 & 3,17 & 0,106 & - \\
\hline & $P^{\prime}-Q^{\prime}$ & 0,10 & 25 & 21,6 & 2,92 & 0,127 & 1,33 \\
\hline \multirow{4}{*}{$\mathrm{B}^{\prime}$} & $A^{\prime}-B^{\prime}$ & 0,25 & 40 & 35,2 & 7,01 & 0,023 & - \\
\hline & $B^{\prime}-O^{\prime}$ & 0,25 & 32 & 27,8 & 10,89 & 0,110 & - \\
\hline & $\mathrm{O}^{\prime}-\mathrm{P}^{\prime}$ & 0,25 & 25 & 21,6 & 3,17 & 0,106 & - \\
\hline & $P^{\prime}-Q^{\prime}$ & 0,10 & 25 & 21,6 & 2,92 & 0,127 & 1,33 \\
\hline \multirow{4}{*}{$\mathrm{C}^{\prime}$} & $A^{\prime}-B^{\prime}$ & 0,20 & 40 & 35,2 & 7,01 & 0,016 & - \\
\hline & $B^{\prime}-O^{\prime}$ & 0,20 & 32 & 27,8 & 10,89 & 0,074 & - \\
\hline & $\mathrm{O}^{\prime}-\mathrm{P}^{\prime}$ & 0,20 & 25 & 21,6 & 3,17 & 0,072 & - \\
\hline & $P^{\prime}-Q^{\prime}$ & 0,10 & 25 & 21,6 & 2,92 & 0,127 & 1,41 \\
\hline \multirow{4}{*}{$\mathrm{D}^{\prime}$} & $A^{\prime}-B^{\prime}$ & 0,20 & 40 & 35,2 & 7,01 & 0,106 & - \\
\hline & $\mathrm{B}^{\prime}-\mathrm{O}^{\prime}$ & 0,10 & 32 & 27,8 & 10,89 & 0,022 & - \\
\hline & $\mathrm{O}^{\prime}-\mathrm{P}^{\prime}$ & 0,10 & 25 & 21,6 & 3,17 & 0,021 & - \\
\hline & $P^{\prime}-Q^{\prime}$ & 0,10 & 25 & 21,6 & 2,92 & 0,127 & 1,51 \\
\hline \multirow{4}{*}{$E^{\prime}$} & $A^{\prime}-B^{\prime}$ & 0,35 & 40 & 35,2 & 7,01 & 0,041 & - \\
\hline & $\mathrm{B}^{\prime}-\mathrm{O}^{\prime}$ & 0,10 & 32 & 27,8 & 10,89 & 0,022 & - \\
\hline & $\mathrm{O}^{\prime}-\mathrm{P}^{\prime}$ & 0,10 & 25 & 21,6 & 3,17 & 0,021 & - \\
\hline & $P^{\prime}-Q^{\prime}$ & 0,10 & 25 & 21,6 & 2,92 & 0,127 & 1,49 \\
\hline
\end{tabular}

Analisando os resultados obtidos para o banheiro 2, percebe-se que as cargas de pressão dinâmica tiveram comportamento análogo às do banheiro 1 onde, devido aos valores inferiores de vazão, todas as combinações apresentaram valores superiores ao obtido pelo Método do Consumo Máximo Provável. Ainda, semelhantemente ao banheiro 1, o maior valor de carga de pressão dinâmica foi encontrado no uso simultâneo dos dois chuveiros, na combinação $\mathrm{D}^{\prime}$. Isso se deve ao fato de que além de apresentar um baixo valor de vazão de projeto, devido ao traçado do sistema, tal aparelho influencia apenas em um trecho que é comum aos dois chuveiros ( $\left.\mathrm{A}^{\prime}-\mathrm{B}^{\prime}\right)$.

Entretanto, a menor pressão não se manteve para a combinação do chuveiro com a pia (combinação E'), e sim para as combinações do chuveiro com o lavatório e com a bacia sanitária (combinações $A^{\prime}$ e $B^{\prime}$ ). A explicação para esse fato é que esses aparelhos interferem em um maior comprimento de trechos que são comuns ao chuveiro (A'-B', $B^{\prime}-\mathrm{O}^{\prime}$ e $\left.0^{\prime}-\mathrm{P}^{\prime}\right)$, que totalizam um comprimento virtual de $21,07 \mathrm{~m}$, tendo, neste caso, um peso maior na geração de perda de carga em relação à vazão combinada do chuveiro e pia com um menor trecho a ser percorrido, apresentando assim um menor valor de carga de pressão dinâmica.

Outro fator que contribuiu para o aumento da perda de carga e, consequentemente, diminuição da carga de pressão dinâmica nas referidas combinações, foi a redução gradual do diâmetro ocorrida na transição entre os trechos A'-B', B'-O' e 0'$\mathrm{P}^{\prime}$, passando de DN $40 \mathrm{~mm}$ para DN $32 \mathrm{~mm}$, e de DN $32 \mathrm{~mm}$ para DN $25 \mathrm{~mm}$, respectivamente. Para efeito de comparação, na combinação E' ocorreu interferência da vazão de projeto da pia apenas no trecho A'-B', que apresenta comprimento virtual de 7,01 m e diâmetro de DN 40 mm. 
Além disso, percebeu-se que os valores de carga de pressão dinâmica das combinações foram maiores para o chuveiro do banheiro 2. Essa superioridade deve-se, principalmente, a um menor valor de perda de carga no registro de pressão localizado à montante do chuveiro, já que o diâmetro utilizado no sub-ramal do chuveiro do banheiro 2 é superior ao utilizado para no sub-ramal do chuveiro do banheiro 1, o que leva a utilizar um coeficiente $\mathrm{K}$ menor, conforme sugere a norma ABNT NBR 15704-1:2011 (ABNT, 2011). Neste estudo foram utilizados os valores máximos de $K$ previsto pela anteriormente citada norma, a fim de se estabelecer cenários mais conservadores quanto ao funcionamento hidráulico. Deste modo, com uma menor perda de carga no registro de pressão, a carga de pressão dinâmica no chuveiro do banheiro 2 se manteve superior em relação ao chuveiro do banheiro 1.

Para a instalação avaliada, ao comparar o trajeto percorrido pela água na tubulação para ambos os chuveiros, tem-se que para o chuveiro do banheiro 1 , o comprimento virtual é de 18,04 m, enquanto para o chuveiro do banheiro 2 é de $24 \mathrm{~m}$. Já em relação ao diâmetro interno, a água percorre por trechos com maiores diâmetros até o chuveiro do banheiro 1 em relação ao chuveiro do banheiro 2, o que leva a uma perda de carga menor. Porém, mesmo assim, o chuveiro do banheiro 1 apresentou maior perda de carga total, justamente devido ao registro de pressão de menor diâmetro utilizado no subramal que liga o ramal até o chuveiro, o qual apresentou uma perda de carga de 0,35 m.c.a., enquanto no sub-ramal que liga o ramal ao chuveiro do banheiro 2 a perda de carga foi de 0,11 m.c.a.

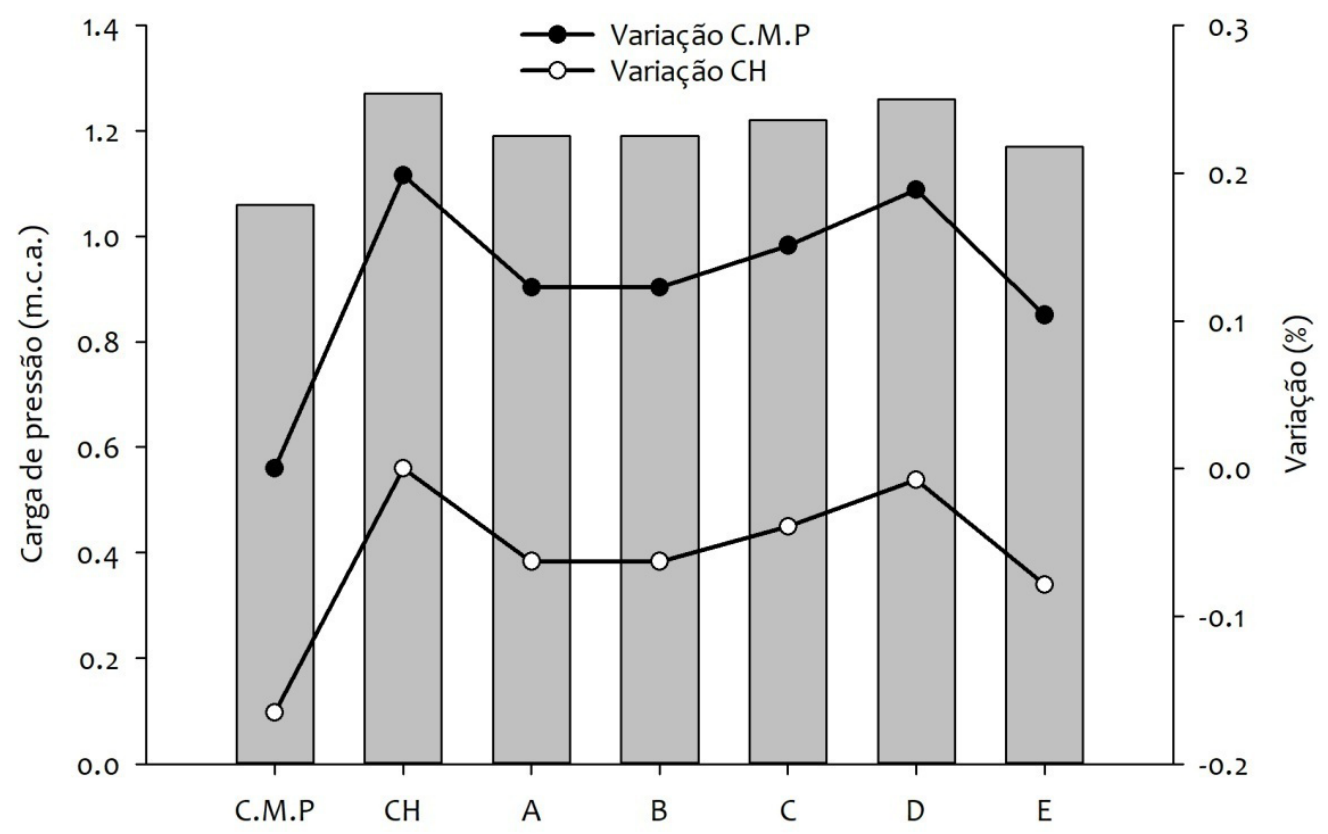

Figura 5. Cargas de pressão dinâmica e suas variações, em porcentagem, para a operação do chuveiro do banheiro 1 em uso simultâneo com outros aparelhos, em relação ao uso isolado do chuveiro com vazão de projeto e ao Método do Consumo Máximo Provável. Legenda: C.M.P = carga de pressão dinâmica obtida para o Método do Consumo Máximo Provável; $\mathrm{CH}$ = carga de pressão dinâmica obtida para o chuveiro operando isoladamente com vazão de projeto; A, B, C, D e E = cargas de pressão dinâmica obtidas para as respectivas combinações; Variação C.M.P. = variação da carga de pressão dinâmica em relação ao obtido para o Método do Consumo Máximo Provável; e Variação $\mathrm{CH}=$ variação da carga de pressão dinâmica em relação ao obtido para o chuveiro operando isoladamente com vazão de projeto. 
Para uma melhor análise do comportamento da carga de pressão dinâmica, na Figura 5 é relacionado as cargas de pressão dinâmica obtidas nas combinações e suas porcentagens de variação em relação ao uso isolado do chuveiro com vazão de projeto e ao Método do Consumo Máximo Provável para todos os casos no chuveiro do banheiro 1.

Nota-se, por meio da Figura 5 que não houve redução da pressão dinâmica (variação negativa) em nenhum dos casos analisados, tendo como base o Método do Consumo Máximo Provável. Tal ocorrido se deve ao fato de que as vazões utilizadas para as combinações de consumo simultâneo e para o chuveiro isolado são menores que as utilizadas no Método do Consumo Máximo Provável, resultando em menores perdas de carga e consequentemente em maiores cargas de pressão dinâmica. Quanto à variação em relação ao uso do chuveiro isolado com vazão de projeto, percebe-se que houve redução da pressão dinâmica (variação negativa) em todas as comparações. Entretanto, nota-se que com exceção da comparação com o Método do Consumo Máximo Provável, que considera uma maior vazão durante a operação do sistema, todas as reduções de cargas pressão dinâmica foram menores do que $10 \%$ do valor de carga de pressão dinâmica do chuveiro funcionando com vazão de projeto, atendendo assim o exigido na norma ABNT NBR 5626:2020 (ABNT, 2020).

Na Figura 6, é relacionado às cargas de pressão dinâmica obtidas nas combinações e suas porcentagens de variação em relação ao uso isolado do chuveiro com vazão de projeto e ao Método do Consumo Máximo Provável para todos os casos no chuveiro do banheiro 2 .

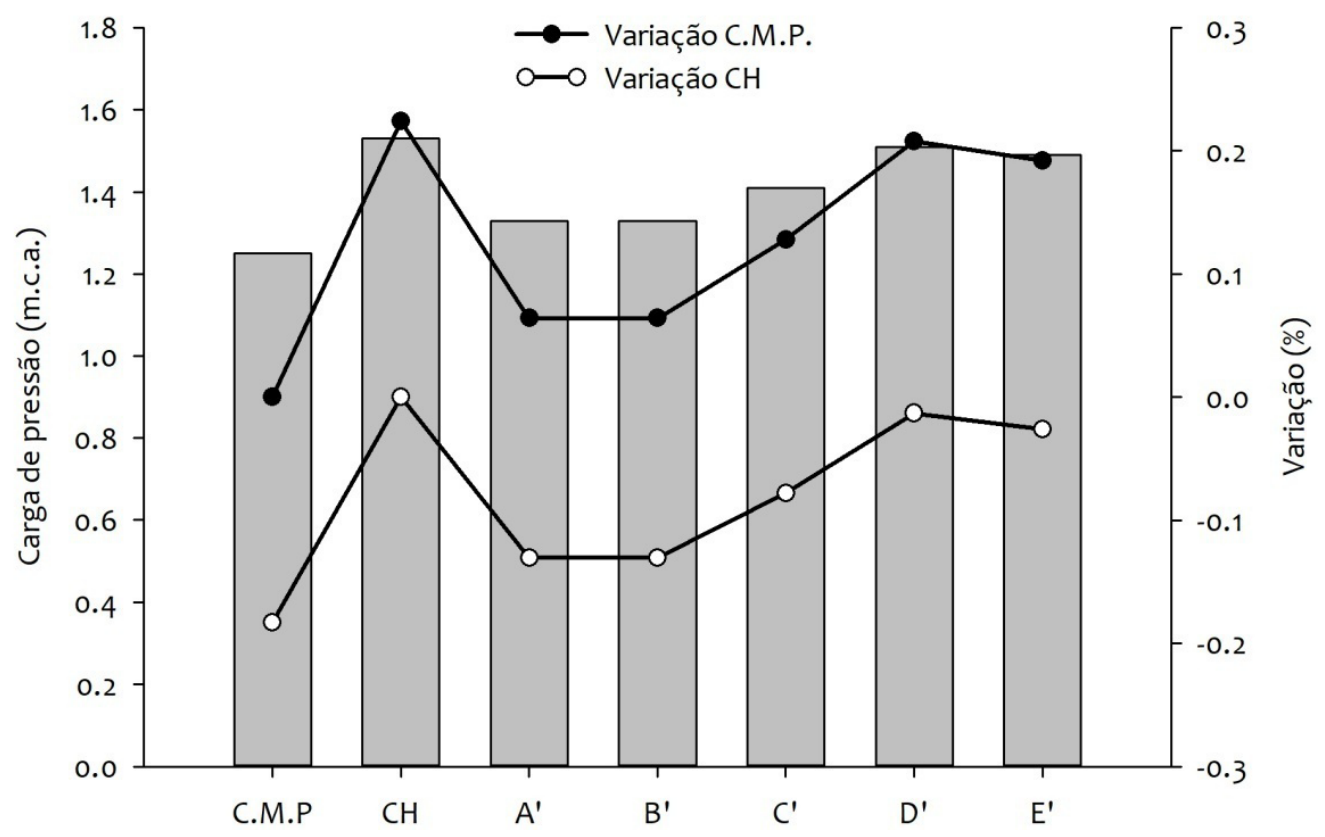

Figura 6. Cargas de pressão dinâmica e suas variações, em porcentagem, para a operação do chuveiro do banheiro 2 em uso simultâneo com outros aparelhos, em relação ao uso isolado do chuveiro com vazão de projeto e ao Método do Consumo Máximo Provável. Legenda: C.M.P = carga de pressão dinâmica obtida para o Método do Consumo Máximo Provável; $\mathrm{CH}$ = carga de pressão dinâmica obtida para o chuveiro operando isoladamente com vazão de projeto; A, B, C, D e E = cargas de pressão dinâmica obtidas para as respectivas combinações; Variação C.M.P. = variação da carga de pressão dinâmica em relação ao obtido para o Método do Consumo Máximo Provável; e Variação $\mathrm{CH}$ = variação da carga de pressão dinâmica em relação ao obtido para o chuveiro operando isoladamente com vazão de projeto. 
De forma análoga ao chuveiro do banheiro 1, no chuveiro do banheiro 2 não houve redução da pressão dinâmica (variação negativa) em nenhum dos casos analisados, tendo como base o Método do Consumo Máximo Provável. Ainda, semelhantemente ao chuveiro do banheiro 1, para a variação em relação ao uso do chuveiro isolado com vazão de projeto, houve redução da pressão dinâmica (variação negativa) em todas as comparações.

Entretanto, diferentemente do ocorrido no banheiro 1, a redução em duas das cinco combinações de uso simultâneo do chuveiro ( $A^{\prime}$ e $B^{\prime}$ ) foram maiores que $10 \%$, ultrapassando o recomendado pela norma ABNT NBR 5626:2020 (ABNT, 2020). Este resultado pode ser explicado pela interferência dessas combinações em todos os trechos da tubulação que ligam o reservatório ao chuveiro, gerando uma maior perda de carga. Nota-se também que da mesma forma, a combinação entre chuveiro e ducha higiênica ( $C^{\prime}$ ) percorre o mesmo trecho das combinações $A^{\prime}$ e B', contudo a vazão de projeto da ducha higiênica é inferior às vazões de projeto da bacia sanitária e do lavatório.

De forma geral, percebe-se que em comparação com o Método do Consumo Máximo Provável em ambos os banheiros, as combinações não provocaram redução maior que $10 \%$ na carga de pressão dinâmica, tampouco houve redução. Já para a comparação com o chuveiro em funcionamento isolado com vazão de projeto, para o banheiro $2 \mathrm{o}$ dimensionamento feito da maneira como era preconizado na norma ABNT NBR 5626:1998 (ABNT, 1998), quando da comparação com a pressão dinâmica estimada para os cenários de funcionamento simultâneo, não atendeu os requisitos da nova edição, exigindo um novo dimensionamento para que se obtenha valores de variação de pressão dinâmica dentro do preconizado. Da mesma maneira que a norma ABNT NBR 5626:2020 (ABNT, 2020), a norma europeia BS EN 806-3 (BSI, 2006) sugere que a carga de pressão em chuveiros seja de $100 \mathrm{kPa}$, contudo, tal norma não faz restrição quanto às reduções decorrentes de usos simultâneos, sendo assim, de mais simples verificação de adequabilidade do Método do Consumo Máximo Provável.

\section{Conclusão}

Após o pré-dimensionamento foi necessário um redimensionamento nos trechos que conduziam a vazão destinada aos chuveiros, para que assim a carga de pressão dinâmica em tais pontos ficasse acima do valor mínimo permitido pela norma ABNT NBR 5626:2020 (ABNT, 2020).

0 chuveiro do banheiro 1 apresentou valores de cargas de pressão dinâmica menores que os do banheiro 2 , devido principalmente à utilização, no último trecho, de um registro de pressão em uma tubulação de diâmetro menor, o que levou a uma maior de perda de carga total na tubulação e, consequentemente, uma menor carga de pressão dinâmica.

Com a utilização das vazões de projeto nas combinações de uso simultâneo do chuveiro com diferentes aparelhos, constatou-se que ao comparar as variações das cargas de pressão dinâmica de cada caso em relação ao obtido por meio do Método do Consumo Máximo Provável, todas sofreram aumento, já que tal método utiliza vazões superiores às utilizadas nas combinações de uso simultâneo deste estudo.

Ao tomar como referência o uso isolado do chuveiro com vazão de projeto, por ser a simulação em que se obteve um maior valor de carga de pressão dinâmica, percebe-se que as variações das cargas pressão dinâmica sofreram uma redução em todos os casos. Contudo, para o chuveiro do banheiro 1 o limite estipulado pela norma ABNT NBR 5626:2020 (ABNT, 2020) não foi excedido e para o chuveiro do banheiro 2 uma redução maior que a preconizada ocorreu para as combinações de uso simultâneo A' e B'.

Portanto, para a edificação estudada, unifamiliar típica, com apenas um pavimento, o Método do Consumo Máximo Provável pôde ser utilizado para o dimensionamento de sistemas prediais de água fria, desde que sejam verificadas as variações das cargas de 
pressão dinâmica nos pontos mais desfavoráveis da edificação. Ainda, para casos em que dois ou mais aparelhos estiverem funcionando simultaneamente com o chuveiro na instalação, o funcionamento segundo o preconizado pela norma ABNT NBR 5626:2020 (ABNT, 2020) pode ser comprometido.

\section{Conflito de interesses}

Os autores declaram não haver conflito de interesses.

\section{Referências}

ABNT - Associação Brasileira de Normas Técnicas. ABNT NBR 5626:1998 - Sistemas prediais de água fria. Rio de Janeiro: ABNT, 1998.

ABNT - Associação Brasileira de Normas Técnicas. ABNT NBR 5626:2020 - Sistemas prediais de água fria e água quente - Projeto, execução, operação e manutenção. Rio de Janeiro: ABNT, 2020.

ABNT - Associação Brasileira de Normas Técnicas. ABNT NBR 15704-1: 2011 - Registro Requisitos e métodos de ensaio. Parte 1: Registros de pressão. Rio de Janeiro: ABNT, 2011.

Azevedo Netto, J. M.; Fernández y Fernández, M. Manual de hidráulica. São Paulo: Blücher, 2015.

Botelho, M. H. C.; Ribeiro Júnior, G. A. Instalações hidráulicas prediais: utilizando tubos plásticos. São Paulo: Blücher, 2014.

BSI - British Standart Institution. BS 6700: Design, installation, testing and maintenance of services supplying water for domestic use within buildings and their curtilages Specification. United Kingdom: BSI, 2006.

BSI - British Standart Institution. BS EN 806-3: Specifications for installations inside buildings conveying water for human consumption - Part 3: Pipe sizing - Simplified method. United Kingdom: BSI, 2006.

Carvalho Júnior, R. Patologias em sistemas hidráulico-sanitários. 2015. Disponível em: <https://www.aea.com.br/blog/patologias-em-sistemas-prediais-hidraulico-sanitarios/>. Acesso em: 19 jul. 2021.

Ilha, M. S. O.; Gnipper, S. F. A. A investigação patológica na melhoria dos sistemas prediais hidráulico-sanitários. Hydro, v. 30, n. 30, p. 60-65, 2009.

Mangalenkar, R. D.; Gumaste, K. S. Residential water demand modelling and hydraulic reliability in design of building water supply systems: A review. Water Supply, v. 21, n. 4, p. 1385-1397, 2021. https://doi.org/10.2166/ws.2021.021

Oliveira, M. F. Análise comparativa entre métodos de dimensionamento de instalações prediais de água fria: Manual e com software comercial. Santa Maria: Universidade Federal de Santa Maria, 2015.(Trabalho de conclusão de curso).

Zanoni, E. B. Análise comparativa entre métodos de dimensionamento de instalações prediais de água fria: manual e com utilização de software. Toledo: Universidade Tecnológica Federal do Paraná, 2018. (Trabalho de conclusão de curso).

Informação da Licença: Este é um artigo Open Access distribuído sob os termos da Licença Creative Commons Attribution, que permite uso irrestrito, distribuição e reprodução em qualquer meio, desde que a obra original seja devidamente citada. 